\title{
Discovering the Context of People With Disabilities: Semantic Categorization Test and Environmental Factors Mapping of Word Embeddings from Reddit
}

Alejandro Garcia-Rudolph ${ }^{1,2,3}, \mathrm{PhD}$; Joan Sauri ${ }^{1,2,3}, \mathrm{PhD}$; Blanca Cegarra ${ }^{1,2,3,4}, \mathrm{MSc}$; Montserrat Bernabeu Guitart ${ }^{1,2,3}$, MD

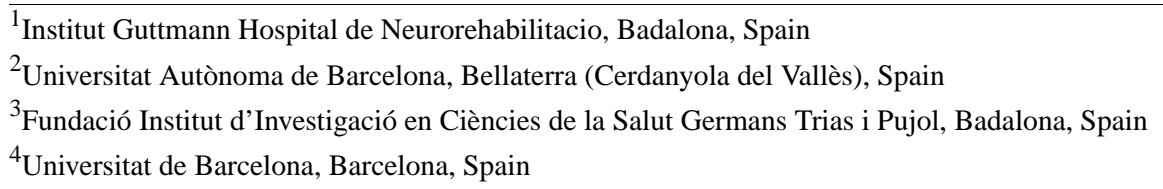

Corresponding Author:

Alejandro Garcia-Rudolph, PhD

Institut Guttmann Hospital de Neurorehabilitacio

Camí de Can Ruti, s / n

Badalona,

Spain

Phone: 34934977700

Email: alejandropablogarcia@gmail.com

\section{Abstract}

Background: The World Health Organization's International Classification of Functioning Disability and Health (ICF) conceptualizes disability not solely as a problem that resides in the individual, but as a health experience that occurs in a context. Word embeddings build on the idea that words that occur in similar contexts tend to have similar meanings. In spite of both sharing "context" as a key component, word embeddings have been scarcely applied in disability. In this work, we propose social media (particularly, Reddit) to link them.

Objective: The objective of our study is to train a model for generating word associations using a small dataset (a subreddit on disability) able to retrieve meaningful content. This content will be formally validated and applied to the discovery of related terms in the corpus of the disability subreddit that represent the physical, social, and attitudinal environment (as defined by a formal framework like the ICF) of people with disabilities.

Methods: Reddit data were collected from pushshift.io with the pushshiftr R package as a wrapper. A word2vec model was trained with the wordVectors $\mathrm{R}$ package using the disability subreddit comments, and a preliminary validation was performed using a subset of Mikolov analogies. We used Van Overschelde's updated and expanded version of the Battig and Montague norms to perform a semantic categories test. Silhouette coefficients were calculated using cosine distance from the wordVectors $\mathrm{R}$ package. For each of the $5 \mathrm{ICF}$ environmental factors (EF), we selected representative subcategories addressing different aspects of daily living (ADLs); then, for each subcategory, we identified specific terms extracted from their formal ICF definition and ran the word2vec model to generate their nearest semantic terms, validating the obtained nearest semantic terms using public evidence. Finally, we applied the model to a specific subcategory of an EF involved in a relevant use case in the field of rehabilitation.

Results: We analyzed 96,314 comments posted between February 2009 and December 2019, by 10,411 Redditors. We trained word2 vec and identified more than 30 analogies (eg, breakfast $-8 \mathrm{am}+8 \mathrm{pm}=$ dinner). The semantic categorization test showed promising results over 60 categories; for example, $\mathrm{s}$ (A relative $)=0.562$, $\mathrm{s}$ (A sport $)=0.475$ provided remarkable explanations for low s values. We mapped the representative subcategories of all EF chapters and obtained the closest terms for each, which we confirmed with publications. This allowed immediate access ( $\leq 2$ seconds) to the terms related to ADLs, ranging from apps "to know accessibility before you go" to adapted sports (boccia). For example, for the support and relationships EF subcategory, the closest term discovered by our model was "resilience," recently regarded as a key feature of rehabilitation, not yet having one unified definition. Our model discovered 10 closest terms, which we validated with publications, contributing to the "resilience" definition. 
Conclusions: This study opens up interesting opportunities for the exploration and discovery of the use of a word2vec model that has been trained with a small disability dataset, leading to immediate, accurate, and often unknown (for authors, in many cases) terms related to ADLs within the ICF framework.

(JMIR Med Inform 2020;8(11):e17903) doi: 10.2196/17903

\section{KEYWORDS}

disability; Reddit; social media; word2vec; semantic categorization; silhouette; activities of daily life; aspects of daily life; context; embeddings

\section{Introduction}

\section{General Background}

Natural Language Processing (NLP) is increasingly being integrated into several application domains. Google AI recently introduced BERT (Bidirectional Encoder Representations from Transformers) [1] to match search queries with more relevant results for optimizing Google searches. Facebook AI also achieved impressive breakthroughs, such as by tackling harmful or improper content by means of Whole Post Integrity Embeddings (WPIE) [2]. Other examples can be found in mobile apps, such as virtual assistants like Amazon's Alexa or Apple's Siri [3]. Application domains range from cultural heritage [4] to the identification of concepts and relationships in a body of research papers [5] or clinical decision support systems [6].

Words that occur in similar contexts tend to have similar meanings. This was likely first formulated in 1954 by Harris [7]. But the most famous statement of this principle came a few years later from linguist JR Firth: "You shall know a word by the company it keeps!" [8].

One of the strongest trends in NLP at the moment is the use of word embeddings, which are vectors whose relative similarities correlate with semantic similarity, building on the ideas of Harris and Firth.

The approval of the International Classification of Functioning, Disability, and Health (ICF) [9] by the World Health Assembly in May 2001 has marked a paradigm shift in the way health and disability are understood and measured [10]. The ICF conceptualizes disability not solely as a problem that resides in the individual but as a health experience that occurs in a context [11].

Disability and functioning are, according to the ICF model, outcomes of interactions between health conditions (diseases, disorders, and injuries) and contextual factors [9].

In spite of both sharing context as a key component, word embeddings have been scarcely applied in the field of disability, to the best of our knowledge.

In this paper, we hypothesize that social media can, indeed, link them. Word embeddings are usually learned from a general-purpose corpus; when it doesn't match the domain's vocabulary (including the same words or using words in the same senses), it is a problem that cannot simply be fixed with a lot of data. More data could just pull word contexts and representations towards generic, rather than domain-specific, values.
Our hypotheses in this paper are the following: (1) Such domain-specific values can be extracted from public domain-specific social media (2) in a sufficient number for the embedding to be relevant to the ICF model and (3) verifiable by sound theoretical semantic tests (4) consistent with state-of-the-art publications and (5) providing actionable knowledge to onfield specialists.

\section{Social Media}

Social media statistics from 2019 show that there are 3.2 billion social media users worldwide, and this number is growing [12].

Recent analyses remark that $42 \%$ of internet users take advantage of social media for health information, $32 \%$ of social media users in the United States share their health care experiences and family's struggle stories, and $29 \%$ search for health information via social media platforms to observe others' experiences with their diseases. Furthermore, $51 \%$ of those who live with a chronic disease have used the internet for information about health topics, such as details of a specific disease, medical procedures, drugs, medical devices, or health insurances [13].

\section{Reddit}

Social media users on platforms such as Reddit [14] tend to sharply contrast with similar groups that participate offline; for instance, people on Reddit are likely to discuss problems that they do not feel comfortable discussing face-to-face [15].

Another reason Reddit was chosen as a data source for this study is that the language of text posts is more structured than on other social media platforms such as Twitter [13].

As of 2019, Reddit's official statistics included 430 million monthly active users, 199 million posts, 1.7 billion comments, and around 14 billion views in a single month [16].

Reddit's core functionality is the sharing of text-based posts with others who may or may not be members of the site. The subforum function allows the creation of designated spaces for users to congregate and interact with each other over a shared interest. These subforums are called subreddits.

\section{This Study}

In the following subsections, we describe the specific characteristics and objectives of this study.

\section{The Disability Subreddit}

The data used for this study were extracted from the disability subreddit (containing news, resources, and perspectives pertaining to individuals with disabilities). It numbers 17,545 subscribers and 17 comments per day [17]. The evolution of 
the number of subscribers since 2013 is shown in Figure 1. The disability subreddit was created on March 12, 2008.

The total number of posts and comments since 2008 are not shown in the Reddit official statistics; the 17 comments per day are, in fact, "the comments received on all its posts in a recent 24 hour measurement period. This number isn't averaged over

Figure 1. The number of subscribers in the disability subreddit by year. time" [17]. A plot of the comments per day during the November 2018-December 2019 period can also be obtained from the official statistics [17] (Multimedia Appendix 1). Therefore, taking as a starting point the total number of subscribers (Figure 1) and the total number of comments during the last year, a rough estimation leads to about 100,000 comments during the 2009-2019 time period.

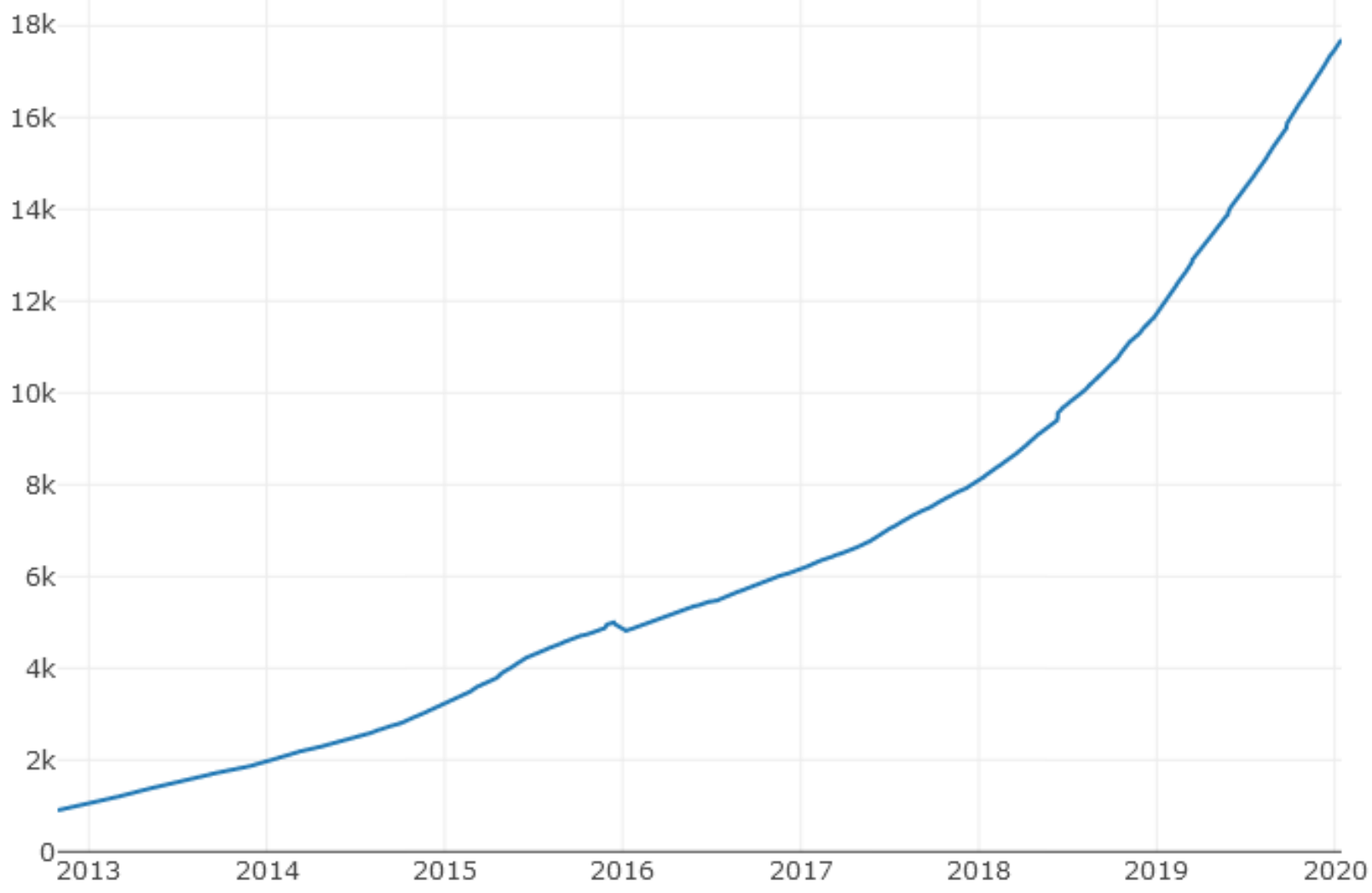

\section{Domain-Specific Values: Environmental Factors}

According to the ICF, contextual factors represent the complete background of an individual's life and living [18]. They include 2 components: environmental factors $(\mathrm{EF})$ and personal factors, which may have an impact on an individual with a health condition and that individual's health and health-related states.

In this study, we focus on the EF section of the ICF; by definition, EF make up the physical, social, and attitudinal environment in which people live and conduct their lives [9]. EF are organized into 5 chapters, each dealing with different and mutually exclusive aspects of the environment: (e1) products and technology, (e2) natural environment and human-made changes to the environment, (e3) support and relationships, (e4) attitudes, and (e5) services, systems, and policies.

\section{Word2vec}

Word2vec is the method used in this study for generating word embeddings. It creates an embedding (ie, numerical representations of words that help capture meaning, semantic relationships, and context) for text by using each word in a corpus to predict the words that usually surround it [19]. It consists of 2 neural network models: Continuous Bag of Words (CBOW) and Skip-gram. In both models, a window of predefined length is moved along the corpus, and in each step, the network is trained with the words inside the window to predict the word in the center of the window based on the surrounding words (CBOW) or to predict the contexts based on the central word (Skip-gram).

Therefore, word2vec creates word embeddings in which the semantic relationships between words are preserved. In this paper, we use the Skip-gram model, which shows better performance in semantic tasks [20].

\section{Small Dataset: Twofold Validation of Word2vec Embedding}

Word2vec methods have a distinct advantage in handling large datasets and have been trained with billions of tokens, as shown in the Google archive of the original Mikolov paper [21] (eg, the latest Wikipedia dumps 3 billion words, or the "one-billion-word language-modeling benchmark").

As it is a prediction-based model, it might be reasonable to expect that word $2 \mathrm{vec}$ will produce very low-quality embeddings when trained with a small corpus.

Nevertheless, based on the high specificity of the disability subreddit, we hypothesize that the produced embeddings will 
be of high semantic quality. In order to verify this, we will follow a twofold validation.

First, we will apply the semantic categorization test in order to measure the word2vec model's capabilities of representing semantic categories (such as vegetables, countries, fruits, and clothes). The original test (the Battig and Montague norm [22]) is composed of 53 categories with 10 words each. In order to measure how well the word $i$ is grouped in relation to the other words in its semantic category, we will use silhouette coefficients [23].

Second, for each EF chapter (e1-e5), we will map meaningful word embeddings to representative categories of each chapter and refer to relevant publications to confirm their practical value.

\section{Study Objectives}

Specific aspects of disability (eg, depression) have been studied on social platforms such as Twitter [24] or support groups for Autism Spectrum Disorder on Facebook [25]. However, to our knowledge, no published study has examined social media content related to the environmental factors that make up the physical, social, and attitudinal environment in which people with disabilities live and conduct their lives.

Therefore, in this study, we aim to do the following: (1) extract all comments and submissions from the disability subreddit during the period under study (2009-2019); (2) train a word2vec model using disability subreddit comments as a training set, performing a preliminary validation using a subset of the original Mikolov paper analogies; (3) perform a semantic categorization test using an updated and expanded version of the Battig and Montague norm, with 65 categories; for each category, compute the silhouette coefficient of the model; (4) select representative subcategories addressing different aspects of daily life for each ICF chapter (e1-e5); for each subcategory, identify specific terms, $t_{i}, t_{j}$, extracted from their formal ICF definition, and run the word2 vec model to generate the nearest semantic terms to $t_{i}$, and $t_{j}$; validate the obtained nearest semantic terms using relevant published literature; and (5) apply the results to a specific subcategory of a chapter involved in a relevant use case in the field of rehabilitation directly involved in daily living.

\section{Methods}

\section{Data Collection}

Reddit data were collected from pushshift.io [26] by the pushshift.io API (application programming interface). pushshift.io is a website that stores all publicly available Reddit submissions and comments, allowing researchers to collect and share Reddit datasets for research purposes, with extensive publications in related research (eg, Lama et al [27]). In this paper, we used the pushshiftr $\mathrm{R}$ package as a wrapper for the pushshift.io API [26].

\section{Word2vec}

For training the word2vec model, we used the wordVectors R package [28]. It implements the original C code for word2vec [20].

\section{Semantic Distances}

Given a vectorial representation of 2 words, their semantic similarity (S) was calculated using the cosine similarity measure between their respective vectorial representation, $\mathrm{S}(\mathrm{v} 1, \mathrm{v} 2)$. The semantic distances between 2 words, d(v1; v2), was calculated as 1 minus the semantic similarity, $\mathrm{d}(\mathrm{v} 1 ; \mathrm{v} 2)=1-\mathrm{S}(\mathrm{v} 1$; v2) [28].

\section{Semantic Categorization Test}

In this test, we measured the capabilities of the model to represent the semantic categories based on the Battig and Montague category norms, an invaluable tool for researchers in many fields, with a recent literature search revealing their use in over 1600 publications in more than 200 different journals [29].

In this study, we use Van Overschelde's [29] updated and expanded version of the Battig and Montague norms (expanded from 56 to 70 semantic categories).

In order to measure how well a word $i$ is grouped in relation to the other words in its semantic category, we used the silhouette coefficients, $s(i)$.

$$
s(i)=\frac{b(i)-a(i)}{\max \{a(i), b(i)\}}
$$

$a(i)$ is the mean distance of word $i$ with all other words within the same category, and $b(i)$ is the minimum mean distance of word $i$ to any words within another category (ie, the mean distance to the neighboring category). In other words, silhouette coefficients measure how close a word is to other words within the same category compared to words of the closest category [23].

\section{Results}

\section{Sample Description}

Data were collected from 20,344 submissions and 96,314 comments from the disability subreddit.

Total comments were posted by 10,411 Redditors, and the total submissions were posted by 9658 Redditors.

The total number of different Redditors that have posted a submission or a comment is 15,072 .

Considering that Reddit moderators remove a percentage of submissions (eg, for not following the Reddit posting guidelines), the number of different Redditors $(15,072)$ is quite close to the 17,545 subscribers presented in Figure 1.

The first comment on the disability subreddit included in this analysis was published in February 2009, and the last was published in December 2019.

Note that these data were publicly accessible on Reddit and that no personally identifiable information is included in this study. The dataset is publicly available per request.

This paper focuses on the analysis of the 96,314 comments; Multimedia Appendix 1 presents further details on the retrieved data. For example, the most common 10 words are people (appearing 4231 times), disability (3619), time (3418), disabled (2479), feel (2457), lot (2433), person (2264), life (2162), day 
(1841) and job (1811). Multimedia Appendix 1 also features a plot of the percentage of comments containing specific words (eg, anger, hope, change, education) by year, the fastest-growing words, and the words with the steepest increase in past years.

\section{word2vec}

We then ran the train_word2vec function of the wordVectors $\mathrm{R}$ package to train the model, with the following parameters: vectors $=200, \quad$ threads $=4, \quad$ window $=12, \quad$ iter $=5$, negative_samples $=0$.

We performed a preliminary validation using a reduced subset of the original Mikolov paper analogies [20]. We selected only some of those that might fit in our context; therefore, we did not include, for example, the well-known analogy

king-man+woman=queen

but we obtained promising results in a variety of analogies, such as

brother-sister+husband=wife $(0.352)$ (cosine distance

is shown in brackets)

This means that if, for our trained model, we execute the nearest_to function as follows:

nearest_to(model[["brother"]]-model[[“sister"]]+model[["husband"]],5)

then we obtain "wife" as one of our top 5 nearest terms. We obtained several of them, with promising results. For example:

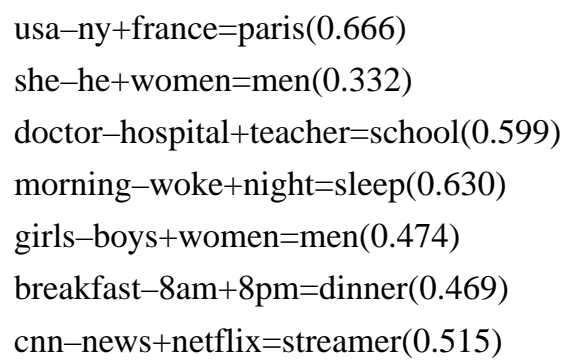

\section{Semantic Categorization Test}

For the first 5 words of each of the first 65 semantic categories of the updated version of the Battig and Montague norm [29], we calculated the silhouette coefficients. The complete list of the 325 words and silhouette calculation is presented in Multimedia Appendix 2.

Multimedia Appendix 3, which shows the silhouette coefficients for the first 60 semantic categories, displays promising results. The numbers identifying each category are those presented in the original norm; for example, the first row is " 3 . A relative" because this is the third semantic category presented in the norm.

When analyzing the lower silhouette scores, we identified remarkable reasons for the miscategorization of the terms. For example, regarding the first semantic category, "1. A precious stone," we selected $i=$ diamond, and our model identified the category "43. A vegetable." To be closer to $i$ than category 1, we tried to find out why broccoli is closer to diamond than, for example, a ruby, and we found that diamond is a well-known class of broccoli. Similar explanations can be found for the other miscategorizations; for example, for category "59. A liquid," we obtained $a(i)=0.490$ for $i=$ water, but $b(i)$ is lower $[b(i)=0.381]$ because it is obtained for category " 38 . A non-alcoholic beverage." This is because water is the first term in category 38 and is included in the mean distance calculation for $b(i)$, (distance $=0)$, but it is not included in the calculation of $a(i)$ because the term $i$ is not included in any calculation of $a(i)$ (Multimedia Appendix 2). The same situation occurs with the category "20. An alcoholic beverage."

It is important to note the lowest silhouette we obtained, $s(i)=-0.645$, for $i=$ tuna in the " 52 . A fish" category (Multimedia Appendix 3). In this case, $b(i)$ is remarkably lower because the distances to category " 43 . A vegetable" are lower for all words in the category. This means that our model finds a closer relation between, for example, broccoli or lettuce with tuna (a healthy diet) than between tuna and shark, bass, beta, or cod, which are very difficult to relate only to fish names.

In spite of obtaining some low silhouette codes, the detailed analysis led to promising results. Table 1 shows the algebraic operations (eg, food+cholesterol) that we used to focus the closest terms onto the specific ICF subcategories, avoiding the miscategorization problems. 
Table 1. Products and technology (e1).

\begin{tabular}{|c|c|}
\hline $2^{\text {nd }}$ order code, $3^{\text {rd }}$ order code, and algebraic operations & Closest terms (cosine distance) \\
\hline \multicolumn{2}{|l|}{ e110 food and drugs } \\
\hline \multicolumn{2}{|l|}{ e1100 food } \\
\hline food+cholesterol & $\begin{array}{l}\text { fruits }(0.347) \text {, veggie }(0.372) \text {, wheat }(0.382) \text {, broccoli }(0.413) \text {, } \\
\text { oatmeal }(0.413)\end{array}$ \\
\hline celiac+analysis & gluten $(0.462)$, mitochondrial $(0.510)$, coronary $(0.513)$, psoriasis $(0.519)$, genetic $(0.526)$ \\
\hline \multicolumn{2}{|l|}{ e1101 drugs } \\
\hline drug+medicinal & $\begin{array}{l}\operatorname{marijuana}(0.342), \text { opioid }(0.348), \text { thc }^{\mathrm{a}}(0.433) \text {, benzos }(0.498), \operatorname{cbd}^{\mathrm{b}}(0.451) \text {, well- } \\
\text { butrin }(0.504)\end{array}$ \\
\hline \multicolumn{2}{|l|}{ e115 assistive products } \\
\hline \multicolumn{2}{|l|}{ e1151 specially designed } \\
\hline voice+controlled & $\operatorname{siri}(0.400)$, dragon(0.406), alexa(0.437) \\
\hline speech+app & voiceover( $(0.366)$, speechyfy $(0.374)$, zoomtext $(0.386)$ \\
\hline \multicolumn{2}{|l|}{ e120 mobility transportation } \\
\hline \multicolumn{2}{|l|}{ e1201 indoor outdoor } \\
\hline motor+outdoor+power & $\begin{array}{l}\text { powerchair }(0.465) \text {, lightest }(0.477) \text {, reclining }(0.482) \text {, Invacare }(0.502) \text {, wijits }(0.506) \text {, } \\
\text { pushrims }(0.501)\end{array}$ \\
\hline transfer+indoor+device & hoist $(0.434)$, stow $(0.439)$, hoyer (0.449), ultralight $(0.497)$ \\
\hline taxi+wheelchair & uber (0.396), lyft (0.406), paratransit $(0.420)$ \\
\hline \multicolumn{2}{|l|}{ e140 culture recreation and sport } \\
\hline \multicolumn{2}{|l|}{ e1401 adapted equipment } \\
\hline adapted+sport & kayaking $(0.389)$, archery $(0.401)$, boccia $(0.437)$, \\
\hline \multicolumn{2}{|l|}{ e150 routing } \\
\hline \multicolumn{2}{|l|}{ e1501 outdoor wayfinding } \\
\hline wheelchair+apps & ableroad $(0.541)$, wheelmap $(0.551)$ \\
\hline
\end{tabular}

athc: tetrahydrocannabinol.

bcbd: cannabidol.

\section{Mapping to the ICF's Environmental Factors}

Table 1 presents the closest terms to the representative subcategories of the products and technology chapter of the Environmental Factors. We present specific terms (eg, food and cholesterol, as illustrative examples) to show the potential of the model to discover relevant terms. In order to validate the results from Table 1, we went through the closest terms for each subcategory and identified recent publications and evidence.

The first terms for e1100, fruits and vegetables (veggie), have been extensively reported to be related to LDL (low-density lipoprotein) cholesterol [30], as well as wheat, oat [31], and broccoli [32].

We then considered other aspects related to food [eg, celiac disease (CD)]; we obtained gluten as the closest result, followed by mitochondrial (eg, reported by Picca et al [33]). The prevalence of the third term, coronaryartery disease (CAD), increases nearly twofold in patients with $\mathrm{CD}$, as reported by Gajulapalli et al [34].
Ungprasert et al [35] demonstrated a significantly higher risk of $\mathrm{CD}$ among patients with psoriasis (fourth closest term) as well as genetic factors [36].

In relation to e1101 (drugs) cannabis, there is an increasing interest in the medical use of it (eg, in chronic pain, which is very common in disability) [37].

In relation to assistive products (eg, related to speech and voice), several state-of-the-art solutions were retrieved, such as Speechify, Zoomtext, Voiceover, Siri, Dragon, and Alexa.

In relation to e120, mobility transportation, desirable properties for outdoor transportation were retrieved, such as lightest or reclining, as well as top product providers (eg, Invacare and wijits). For indoor transportation, the closest terms were hoist, stow, and hoyer, as opposed to powerchair, which was retrieved for outdoors.

We then explored transportation services (taxi+wheelchair), and the closest terms were uber, lyft, and paratransit. It is important to remark that Uber [38] and Lyft provide specific disability policies [39]. 
In relation to e140, culture recreation and sport, several paralympic well-known ports emerged, such as kayaking or archery. Another close term was boccia, which is another (but perhaps less popular) Paralympic sport [40].

Finally, in relation to e1501, outdoor wayfinding, the wheelchair+app operation retrieved ableroad [41] and wheelmap [42] as closest terms.

Multimedia Appendix 2 includes a similar analysis for chapters e2-e5.

\section{Rehabilitation Use Case: Resilience (ICF e398 Subcategory)}

As shown in Multimedia Appendix 2, for the e398 support and relationships subcategory, the closest term identified by our model is resilience. Resilience has been recently regarded as a key feature of rehabilitation and living life well following a disability [43]. Evidence shows that resilience-based skills have multiple benefits once applied to people's lives (eg, a carry-over effect to other life domains). People do not have to be born resilient to become resilient; it can be improved with intentional practice [44].

It is unclear how well resilience or strategies to cultivate resilience are currently promoted as a component of rehabilitation programs. Furthermore, it does not yet have one unified definition, and research scholars have not decided on one specific understanding of what resilience means. Understanding resilience is an important component of building resilience [43].

Therefore, we used our model to identify its closest terms, and then we linked those terms to relevant publications. This is proposed as a straightforward example of how it can contribute to the understanding of a relevant term in rehabilitation. Maturity, compassion, anger, resentment, grief, insecurity, contentment, and resenting were identified by our model as the closest terms to resilience. Resilience and maturity have been extensively reported on (eg, by Davies et al [45]). The practice of compassion has been highlighted as an "essential component in nurturing resilience" [46]. As reported by Baldachino et al [47], the inability to cope effectively with anger may negatively impact a patient's physical and psychological well-being in the realm of resilience. Designing evidence-based interventions aimed at decreasing the negative impact of anger on resilience can be advanced by examining the potential mediation effect between anger and resilience [48].

As noted by Howard and Meichenbaum [49], resentment is a way of undermining resilience; resentment is a form of chronic, deep-seated anger. Holding onto resentment and not letting it go can have deleterious health effects and undermine the development of resilience. In relation to grief, it has also been related to resilience (eg, Bonano et al [50]).

As was presented in the EU Social Insecurities and Resilience Report [51], people who have low levels of social insecurities more often report high levels of resilience. In recent psychological research [52], contentment was also directly associated with both resilience and life satisfaction and mediated the relationship between these 2 aspects of well-being. Details and further analysis is presented in Multimedia Appendix 1.

\section{Discussion}

\section{Principal Findings}

In this paper, we proposed social media as the link between word embeddings and the ICF's environmental factors in a General Public License (GPL) framework (R-3.5.1). We applied a set of publicly available $\mathrm{R}$ libraries for collecting, model training, and analyzing Reddit public data. We trained a word2vec model using a small dataset and obtained encouraging results in king-queen-type analogies. Further, we obtained remarkable results in a standardized semantic categorization test. When mapping the discovered closest terms to representative subcategories of all ICF environmental factors, we verified them with scientific publications.

The obtained results open up interesting opportunities for exploration. Similarity isn't just a way of finding the nearest words; it is also a way of extracting items of a single class in every environmental factor that makes up the physical, social, and attitudinal environment in which people live and conduct their lives, ranging from apps "to know accessibility before you go" to adapted sports (boccia). Therefore, it can be thought of as a form of topic modeling; however, rather than letting the algorithm choose a fixed number of topics, it gives us the option of choosing the specific term (such as resilience) and how expansive we want the explored space to be.

Medical professionals are currently being encouraged to participate in social media, as remarked upon by Stukus [53]; even if a health care professional is not interested in engaging in social media, they must still be aware of the information people may be encountering online in order to provide anticipatory guidance in the clinical setting [47]. Therefore, this study also intends to be a step in that direction.

\section{Limitations}

The collected sample was not intended to be either representative or a comprehensive set of all comments posted by all persons with disabilities during the period under study. It includes all comments posted in Reddit's disability subreddit; we did not include comments from other subreddits addressing specific disability causes (eg, stroke) because, in this study, we follow the approach of studying disability in general (instead of any specific group or etiology), an approach also adopted in other recent literature reviews (eg, Hästbacka et al [54]). In addition, the ICF is grounded in the principle of universality, namely, that functioning and disability are applicable to all people, irrespective of health condition. The ICF is committed to the principle of parity, which states that functional status is not determined by background etiology or, in particular, by whether one has a physical rather than mental health condition [9]

Furthermore, including only 93,614 comments allowed us to verify our hypotheses.

We did not include submissions in our analysis; we collected them (20,344 in total), but we did not use them for model training because only 2745 of them were labeled as "questions" 
while 1256 were labeled "articles and news," 438 of them were labeled "videos," and 13,570 were not labeled.

As presented in Multimedia Appendix 1, under the total number of comments by year, almost $80 \%$ of all comments took place during 2016-2019; therefore, the results of this study are weighted more strongly toward the recent years (2016-2019) rather than the early years (2009-2015) of the Disability subreddit.

Other relevant limitations to our study are related to the geographic location, spatial trajectory, or the time of day on which a comment was posted. As noted by Padilla et al [55] and Gore et al [56], such factors are relevant in social media. Spatiotemporal aspects are not controlled in our study; however, Reddit is most popular in the United States, with American users (representing 54\% of Reddit's user base) far outnumbering those from other countries. Second to the United States, the UK represents the next highest share of Reddit traffic, at $8 \%$, with Canada rounding out third, at $6.4 \%$. Reddit is most popular amongst young adults between the ages of 25-34 years, which make up more than half of the site's users. Nevertheless, Reddit still draws in a large number of middle-aged users. A 2016 study found that people between the ages of 30-49 years accounted for $33 \%$ of the site's users, indicating that Reddit is a viable platform for reaching both young and middle-aged adults. Reddit is particularly popular among males, who make up more than two-thirds of the site's users [16].

\section{Comparison with Prior Work}

In medical applications, word2vec has recently been applied to larger datasets, such as 641,279 French health-related documents produced in a professional context (Dynomant et al [57]), 880,165 papers in a biomedical publication venue (Feng et al [58]), 1,451,413 abstracts for Adverse Drug Event Discovery Using Biomedical Literature (Tafti et al [59]), and 1,749,870 reviews in Online Doctor Reviews [60].

Reddit has recently been used as a data source for studies in chronic diseases, such as Foufi et al's [13] analysis of 17,624 text posts for entity and relation extraction, employing the PKDE4J tool. Sharma et al [61] performed a qualitative analysis of Reddit discussions regarding motivations and limitations associated with vaping among people with mental illness, a thematic analysis that included 3263 comments from 133 discussion threads.
In a small corpus $(37,000$ and 140,000 documents) using a semantic categorization test, word2vec was applied in analyzing and disambiguating the content of dreams [62]; this research field addresses questions such as "how do gender, cultural background, and waking-life experiences shape dream content?".

Facebook groups, discussion forums, and chat rooms were recently analyzed [63] to explore and compare the interactions and connections among online support groups to better understand how people with disabilities were utilizing different social networks to facilitate communication interchange. They concluded that through participation on different platforms, persons with disabilities are able to provide and receive social support in various ways, without the barriers and constraints often experienced by this population.

Our approach is completely different in that we provide a tool for discovering terms of interest (in this paper, we applied it to the ICF environmental factors, but it could also be applied to, for example, the ICF's body functions and structures). For example, regarding e5 services and associations, in using the 3 terms hear+association +kid, we obtained depaul as one of the closest results. The DePaul School for Hearing and Speech teaches children who are deaf or hard of hearing to listen and speak without sign language [64]. Another obtained close term is saskatoon, a new early learning pilot program in Saskatoon that impacts preschoolers who are deaf or hard of hearing by breaking down communication barriers [65].

Therefore, it can be addressed to a wide variety of involved stakeholders besides people with disabilities themselves, such as informal caregivers, health care professionals, and private or public associations.

\section{Conclusions}

This study explored the ability of word2vec to extract the main factors affecting the lives of people with disabilities within the ICF framework from a small dataset, showing promising results.

Our results open up interesting opportunities for exploration and discovery. Similarity is revealed as not only a way of finding the nearest words but also a way of extracting out items related to specific elements. Therefore, it can be thought of as a form of topic modeling, where users can focus on a particular term in-breath or in-depth.

\section{Acknowledgments}

This research was partially funded by PARTICIPA Barriers and Facilitators to Social Participation and PRECISE4Q Personalised Medicine by Predictive Modeling in Stroke for Better Quality of Life H2020, grant number 777107.

\section{Authors' Contributions}

AGR and JSR conceived the study. AGR, JSR, and BCR collected, selected, and cleaned the data. AGR and BCD analyzed the data. AGR and BCD drafted the initial manuscript. JSR and MBG revised the manuscript critically for important intellectual content and approved the final manuscript. AGR, JSR, and MBG received funding for the study.

\section{Conflicts of Interest}

None declared. 


\section{Multimedia Appendix 1}

Word trends, closest terms, environmental factors, and resilience.

[DOCX File, 212 KB-Multimedia Appendix 1]

\section{Multimedia Appendix 2}

Semantic Categorization Test.

[XLSX File (Microsoft Excel File), 427 KB-Multimedia Appendix 2]

\section{Multimedia Appendix 3}

The 60 semantic categories ordered by silhouette value.

[DOCX File, 13 KB-Multimedia Appendix 3]

\section{References}

1. Clark K, Khandelwal U, Levy O, Manning CD. What Does BERT Look at? An Analysis of BERT's Attention. : The Association for Computational Linguistics; 2019 Presented at: The BlackboxNLP Workshop on Analyzing and Interpreting Neural Networks for NLP at ACL 2019; August 2019; Florence, Italy. [doi: 10.18653/v1/w19-4828]

2. MacAvaney S, Yao HR, Yang E, Russell K, Goharian N. Hate speech detection: Challenges and solutions. PLOS ONE 2019;14 (8) [FREE Full text] [doi: 10.1371/journal.pone.0221152]

3. Ni Loideain N, Adams R. From Alexa to Siri and the GDPR: The Gendering of Virtual Personal Assistants and the Role of EU Data Protection Law. SSRN Journal 2018. [doi: 10.2139/ssrn.3281807]

4. Sporleder C. Natural Language Processing for Cultural Heritage Domains. Language and Linguistics Compass 2010;4(10). [doi: $10.1111 /$ j.1749-818x.2010.00230.x]

5. Diallo S, Gore R, Padilla J, Lynch C. An Overview of Modeling and Simulation using Content Analysis. Scientometrics 2015. [doi: 10.1007/s11192-015-1578-6]

6. Demner-Fushman D, Chapman WW, McDonald CJ. What can natural language processing do for clinical decision support? J Biomed Inform 2009 Oct 26;42(5):760-772 [FREE Full text] [doi: 10.1016/j.jbi.2009.08.007] [Medline: 19683066]

7. Harris ZS. Distributional Structure. New York: Verlag; 1954.

8. Firth JR. A synopsis of linguistic theory 1930-1955. In Studies in Linguistic Analysis. Oxford: Palmer; 1957.

9. World Health Organization Geneva. Towards a Common Language for Functioning, Disability and Health. The International Classification of Functioning, Disability and Health. 2002. URL: https://www.who.int/classifications/icf/icfbeginnersguide. pdf [accessed 2020-01-10] [WebCite Cache ID www.who.int/classifications/icf/icfbeginnersguide.pdf]

10. Kostanjsek N. Use of The International Classification of Functioning, Disability and Health (ICF) as a conceptual framework and common language for disability statistics and health information systems. BMC Public Health 2011 May 31;11 Suppl 4(2-3):S3-162 [FREE Full text] [doi: 10.1186/1471-2458-11-S4-S3] [Medline: 21624189]

11. Quah SR, editor. International Encyclopedia of Public Health, 2nd Ed. Cambridge: Academic Press; Nov $03,2016$.

12. Oberlo. 10 Social Media Statistics You Need to Know. URL: https://www.oberlo.com/blog/social-media-marketing-statistics [accessed 2020-01-10] [WebCite Cache ID www.oberlo.com/blog/social-media-marketing-statistics]

13. Foufi V, Timakum T, Gaudet-Blavignac C, Lovis C, Song M. Mining of Textual Health Information from Reddit: Analysis of Chronic Diseases With Extracted Entities and Their Relations. J Med Internet Res 2019 Jun 13;21(6) [FREE Full text] [doi: $10.2196 / 12876]$ [Medline: 31199327$]$

14. reddit. 2002. URL: www.reddit.com [accessed 2020-01-10] [WebCite Cache ID www.reddit.com]

15. Johnson GJ, Ambrose PJ. Neo-tribes: the power and potential of online communities in health care. Commun. ACM 2006 Jan;49(1):107-113 [FREE Full text] [doi: 10.1145/1107458.1107463]

16. Reddit stats. URL: https://redditblog.com/2019/12/04/reddits-2019-year-in-review/ [accessed 2020-01-10] [WebCite Cache ID https://redditblog.com/2019/12/04/reddits-2019-year-in-review/]

17. Disability subreddit. URL: https://subredditstats.com/r/disability [accessed 2020-01-10] [WebCite Cache ID https://subredditstats.com/r/disability]

18. Grotkamp S, Cibis W, Nüchtern E, von Mittelstaedt G, Seger W. Personal Factors in the International Classification of Functioning, Disability and Health: Prospective Evidence. The Australian Journal of Rehabilitation Counselling 2012 Jul 04;18(1):1-24. [doi: 10.1017/jrc.2012.4]

19. Allem JP, Dharmapuri L, Unger JB, Cruz TB. Characterizing JUUL-related posts on Twitter. Drug Alcohol Depend 2018 Sep 01;190:1-5 [FREE Full text] [doi: 10.1016/j.drugalcdep.2018.05.018] [Medline: 29958115]

20. Mikolov T, Corrado G, Chen K, Dean J. Efficient Estimation of Word Representations in Vector Space. In: Proceedings of the International Conference on Learning Representations. 2013 Presented at: International Conference on Learning Representations; May 2nd to May 4th 2013; Scottsdale, Arizona p. 1-12.

21. Google word2vec. URL: https://code.google.com/archive/p/word2vec/ [accessed 2020-01-10] [WebCite Cache ID https://code.google.com/archive/p/word2vec/] 
22. Battig WF, Montague WE. Category norms of verbal items in 56 categories A replication and extension of the Connecticut category norms. Journal of Experimental Psychology 1969 Jun 13;80(3, Pt.2):1-46. [doi: 10.1037/h0027577]

23. Rousseeuw PJ. Silhouettes: A graphical aid to the interpretation and validation of cluster analysis. Journal of Computational and Applied Mathematics 1987 Nov 13;20(6):53-65. [doi: 10.1016/0377-0427(87)90125-7]

24. Leis A, Ronzano F, Mayer MA, Furlong LI, Sanz F. Detecting Signs of Depression in Tweets in Spanish: Behavioral and Linguistic Analysis. J Med Internet Res 2019 Jun 27;21(6):e14199-e14101 [FREE Full text] [doi: 10.2196/14199] [Medline: 31250832]

25. Mustafa HR, Short M, Fan S. Social Support Exchanges in Facebook Social Support Group. Procedia - Social and Behavioral Sciences 2015 May;185:346-351. [doi: 10.1016/j.sbspro.2015.03.449]

26. pushshift.io: API Documentation. URL: https://pushshift.io/api-parameters/ [accessed 2020-01-10] [WebCite Cache ID https://pushshift.io/api-parameters/]

27. Lama Y, Hu D, Jamison A, Quinn SC, Broniatowski DA. Characterizing Trends in Human Papillomavirus Vaccine Discourse on Reddit (2007-2015): An Observational Study. JMIR Public Health Surveill 2019 Mar 27;5(1):e12480-e12113 [FREE Full text] [doi: 10.2196/12480] [Medline: $\underline{\text { 30916662] }}$

28. wordVectors. URL: https://github.com/bmschmidt/wordVectors [accessed 2020-01-10] [WebCite Cache ID https://github.com/bmschmidt/wordVectors]

29. Van Overschelde J, Rawson K, Dunlosky J. Category norms: An updated and expanded version of the Battig and Montague (1969) norms. Journal of Memory and Language 2004 Apr;50(3):289-335. [doi: 10.1016/j.jml.2003.10.003]

30. Djoussé L, Arnett DK, Coon H, Province MA, Moore LI, Ellison RC. Fruit and vegetable consumption and LDL cholesterol: the National Heart, Lung, and Blood Institute Family Heart Study. Am J Clin Nutr 2004 Feb;79(2):213-217. [doi: 10.1093/ajcn/79.2.213] [Medline: 14749225$]$

31. Momenizadeh A, Heidari R, Sadeghi M, Tabesh F, Ekramzadeh M, Haghighatian Z, et al. Effects of oat and wheat bread consumption on lipid profile, blood sugar, and endothelial function in hypercholesterolemic patients: A randomized controlled clinical trial. ARYA Atheroscler 2014 Sep;10(5):259-265 [FREE Full text] [Medline: 25477983]

32. Armah CN, Derdemezis C, Traka MH, Dainty JR, Doleman JF, Saha S, et al. Diet rich in high glucoraphanin broccoli reduces plasma LDL cholesterol: Evidence from randomised controlled trials. Mol Nutr Food Res 2015 May 04;59(5):918-926 [FREE Full text] [doi: 10.1002/mnfr.201400863] [Medline: 25851421]

33. Picca A, Riezzo G, Lezza AMS, Clemente C, Pesce V, Orlando A, et al. Mitochondria and redox balance in coeliac disease: A case-control study. Eur J Clin Invest 2018 Feb 04;48(2):1-24. [doi: 10.1111/eci.12877] [Medline: 29243228]

34. Gajulapalli RD, Pattanshetty DJ. Risk of coronary artery disease in celiac disease population. Saudi J Gastroenterol 2017 Sep;23(4):253-258 [FREE Full text] [doi: 10.4103/sjg.SJG_616_16] [Medline: 28721980]

35. Ungprasert P, Wijarnpreecha K, Kittanamongkolchai W. Psoriasis and Risk of Celiac Disease: A Systematic Review and Meta-analysis. Indian J Dermatol 2017;62(1):41-46. [doi: 10.4103/0019-5154.198031] [Medline: 28216724]

36. Farina F, Picascia S, Pisapia L, Barba P, Vitale S, Franzese A, et al. HLA-DQA1 and HLA-DQB1 Alleles, Conferring Susceptibility to Celiac Disease and Type 1 Diabetes, are More Expressed Than Non-Predisposing Alleles and are Coordinately Regulated. Cells 2019 Jul 19;8(7):2020-2001 [FREE Full text] [doi: 10.3390/cells8070751] [Medline: 31331105]

37. Poli P, Crestani F, Salvadori C, Valenti I, Sannino C. Medical Cannabis in Patients with Chronic Pain: Effect on Pain Relief, Pain Disability, and Psychological aspects. A Prospective Non randomized Single Arm Clinical Trial. Clin Ter 2018;169(3):e102-e107 [FREE Full text] [doi: 10.7417/T.2018.2062] [Medline: 29938740]

38. UBER Accessibility. URL: https://www.uber.com/us/en/about/accessibility/ [accessed 2020-01-10] [WebCite Cache ID https://www.uber.com/us/en/about/accessibility/]

39. Lyft Wheelchair policy. URL: https://help.lyft.com/hc/en-us/articles/115012926827-Wheelchair-Policy [accessed 2020-01-10]

40. Sports: Boccia. URL: https://www.disabledsportsusa.org/sport/boccia/ [accessed 2020-01-10]

41. iaccessiblity.com. AbleRoad. URL: https://www.iaccessibility.com/apps/general/index.cgi/product?ID=3 [accessed 2020-01-10]

42. Wheelmap. URL: https://news.wheelmap.org/en/wheelmap-org-750000-places-in-seven-years/ [accessed 2020-01-10]

43. Stuntzner S, Dalton J, Umeasiegbu V, MacDonald A, Mercado F. Resilience and Disability: Consideration and Integration of Resilience Training in Undergraduate Rehabilitation Service Programs. Journal of Applied Rehabilitation Counseling 2018 Dec 01;49(4):5-13. [doi: 10.1891/0047-2220.49.4.5]

44. Newman R. APA's resilience initiative. Professional Psychology: Research and Practice 2005 Jun;36(3):227-229. [doi: 10.1037/0735-7028.36.3.227]

45. Davies M. Disability and Impairment: Working with Children and Families. British Journal of Social Work 2007 Feb 01;38(8):1656-1657. [doi: 10.1093/bjsw/bcn155]

46. Marini I, Milington M, Graf NM. Psychosocial Aspects of Disability, 2nd Ed. New York: Springer Publishing Company; 2017.

47. Baldacchino DR. Student nurses' personality traits and the nursing profession: part 1. Br J Nurs 2012;21(7):419-425. [doi: 10.12968/bjon.2012.21.7.419] [Medline: 22585020] 
48. Wu W, Chang J, Tsai S, Liang S. Assessing Self-concept as a Mediator Between Anger and Resilience in Adolescents With Cancer in Taiwan. Cancer Nursing 2018 Mar 27;41(3):210-217. [doi: 10.1097/NCC.0000000000000512]

49. Meichenbaum D. Roadmap to Resilience: A Guide for Military, Trauma Victims and Their Families. United Kingdom: CROWN HOUSE PUB LTD; Sep 15, 2012.

50. Bonanno G, Wortman C, Lehman D, Tweed R, Haring M, Sonnega J, et al. Resilience to loss and chronic grief: A prospective study from preloss to 18-months postloss. Journal of Personality and Social Psychology 2002;83(5):1150-1164. [doi: 10.1037/0022-3514.83.5.1150]

51. , editor. Social insecurities and resilience. Luxembourg: Publications Office of the European Union; 2018.

52. Gerson MW. Spirituality, Social Support, Pride, and Contentment as Differential Predictors of Resilience and Life Satisfaction in Emerging Adulthood. Psychology 2018 Apr;09(03):485-517. [doi: 10.4236/psych.2018.93030]

53. Stukus DR, Patrick MD, Nuss K. Social Media for Medical Professionals: Strategies for Successfully Engaging in an Online World. New York: Springer; May 13, 2013.

54. Hästbacka E, Nygård M, Nyqvist F. Barriers and facilitators to societal participation of people with disabilities: A scoping review of studies concerning European countries. European Journal of Disability Research 2016 Jul;10(3):201-220. [doi: 10.1016/j.alter.2016.02.002]

55. Padilla J, Kavak H, Lynch C, Gore R, Diallo S. Temporal and spatiotemporal investigation of tourist attraction visit sentiment on Twitter. PLoS One 2018;13(6):e0198857. [doi: 10.1371/journal.pone.0198857] [Medline: 29902270]

56. Gore R, Diallo S, Padilla J. You Are What You Tweet: Connecting the Geographic Variation in America's Obesity Rate to Twitter Content. PLoS One 2015 Sep;10(9):e0133505-e0133565. [doi: 10.1371/journal.pone.0133505] [Medline: 26332588]

57. Dynomant E, Lelong R, Dahamna B, Massonnaud C, Kerdelhué G, Grosjean J, et al. Word Embedding for the French Natural Language in Health Care: Comparative Study. JMIR Med Inform 2019 Jul 29;7(3):e12310-e12926 [FREE Full text] [doi: 10.2196/12310] [Medline: $\underline{31359873}$ ]

58. Feng X, Zhang H, Ren Y, Shang P, Zhu Y, Liang Y, et al. The Deep Learning-Based Recommender System "Pubmender" for Choosing a Biomedical Publication Venue: Development and Validation Study. J Med Internet Res 2019 May 24;21(5):e12957 [FREE Full text] [doi: 10.2196/12957] [Medline: 31127715]

59. Tafti AP, Badger J, LaRose E, Shirzadi E, Mahnke A, Mayer J, et al. Adverse Drug Event Discovery Using Biomedical Literature: A Big Data Neural Network Adventure. JMIR Med Inform 2017 Dec 08;5(4):e51 [FREE Full text] [doi: 10.2196/medinform.9170] [Medline: 29222076]

60. Rivas R, Montazeri N, Le N, Hristidis V. Automatic Classification of Online Doctor Reviews: Evaluation of Text Classifier Algorithms. J Med Internet Res 2018 Nov 12;20(11):e11141 [FREE Full text] [doi: 10.2196/11141] [Medline: 30425030]

61. Sharma R, Wigginton B, Meurk C, Ford P, Gartner CE. Motivations and Limitations Associated with Vaping among People with Mental Illness: A Qualitative Analysis of Reddit Discussions. Int J Environ Res Public Health 2016 Dec 22;14(1):751 [FREE Full text] [doi: 10.3390/ijerph14010007] [Medline: 28025516]

62. Altszyler E, Ribeiro S, Sigman M, Fernández Slezak D. Comparative study of LSA vs Word2vec embeddings in small corpora: a case study in dreams database. Conscious Cogn 2017 Nov;56(7):178-187.

63. Stetten NE, LeBeau K, Aguirre MA, Vogt AB, Quintana JR, Jennings AR, et al. Analyzing the Communication Interchange of Individuals With Disabilities Utilizing Facebook, Discussion Forums, and Chat Rooms: Qualitative Content Analysis of Online Disabilities Support Groups. JMIR Rehabil Assist Technol 2019 Sep 30;6(2):e12667 [FREE Full text] [doi: 10.2196/12667] [Medline: 31573937]

64. DePaul School for Hearing \& Speech. URL: https://www.depaulhearingandspeech.org/ [accessed 2020-01-10]

65. Stillger N. New program in Saskatoon having positive impact on deaf and hard of hearing preschoolers. Global News. 2018 Dec 11. URL: https://globalnews.ca/news/4752539/preschoolers-deaf-hard-of-hearing-saskatoon-regina/ [accessed 2020-01-10] [WebCite Cache ID https://globalnews.ca/news/4752539/preschoolers-deaf-hard-of-hearing-saskatoon-regina/]

\section{Abbreviations}

ADLs: aspects of daily living

API: application program interface

BERT: bidirectional encoder representations from transformers

CBD: cannabidiol

CBOW: continuous bag of words

EF: environmental factors

ICF: International Classification of Functioning Disability and Health

LDL: low-density lipoprotein

NLP: natural language processing

THC: tetrahydrocannabinol

WPIE: whole post integrity embeddings 
Edited by G Eysenbach; submitted 20.01.20; peer-reviewed by $R$ Gore, M Bjelogrlic; comments to author 10.02.20; revised version received 17.04.20; accepted 19.04.20; published 20.11.20

Please cite as:

Garcia-Rudolph A, Saurí J, Cegarra B, Bernabeu Guitart M

Discovering the Context of People With Disabilities: Semantic Categorization Test and Environmental Factors Mapping of Word Embeddings from Reddit

JMIR Med Inform 2020;8(11):e17903

URL: http://medinform.jmir.org/2020/11/e17903/

doi: $\underline{10.2196 / 17903}$

PMID: 33216006

(C)Alejandro Garcia-Rudolph, Joan Saurí, Blanca Cegarra, Montserrat Bernabeu Guitart. Originally published in JMIR Medical Informatics (http://medinform.jmir.org), 20.11.2020. This is an open-access article distributed under the terms of the Creative Commons Attribution License (https://creativecommons.org/licenses/by/4.0/), which permits unrestricted use, distribution, and reproduction in any medium, provided the original work, first published in JMIR Medical Informatics, is properly cited. The complete bibliographic information, a link to the original publication on http://medinform.jmir.org/, as well as this copyright and license information must be included. 